\title{
Surface Magnetization of Gd at the Bulk Curie Temperature
}

\author{
C. Rau \\ Department of Physics, Rice University, Houston, Texas 77251 \\ and \\ M. Robert \\ Department of Chemical Engineering, Rice University, Houston, Texas 77251 \\ (Received 10 November 1986)
}

\begin{abstract}
Electron-capture spectroscopy is used to study the long-ranged surface ferromagnetic order of $\mathrm{Gd}$ in the neighborhood of the bulk Curie temperature $T^{\text {bulk }}$ at which, unlike bulk ferromagnetic order, it does not vanish. The surface magnetization, together with its first temperature derivative, is found to be a continuous function of temperature at $T \mathrm{C}^{\text {ulk }}$ and, as the temperature is varied, the surface magnetization is found to depart linearly from the nonzero value which it assumes at $T^{\text {bulk}}$, with critical exponents $x_{+}=1.00 \pm 0.03\left(T>T \mathcal{R}^{\text {ulk }}\right)$ and $x_{-}=1.00 \pm 0.01\left(T<T^{\text {bulk }}\right)$. These findings are discussed in the light of recent theoretical studies.
\end{abstract}

PACS numbers: $75.50 . \mathrm{Cc}, 75.40 .-\mathrm{s}$

It is now well established that the magnetic properties of surfaces may differ drastically from those of bulk matter to which they are coupled. '

A particularly striking illustration of this fact is provided by the possibility of the coexistence of a magnetically ordered surface phase with a magnetically disordered bulk phase.

This remarkable phenomenon was discovered ${ }^{2}$ in 1981 in $\mathrm{Gd}$, with use of electron-capture spectroscopy, a new method that probes magnetic order at the topmost surface layer. Possibly related observations have more recently been made by spin-polarized photo emission ${ }^{3}$ and spin-polarized low-energy-electron diffraction (SPLEED), ${ }^{4}$ techniques which, however, probe simultaneously the first few surface layers rather than only the topmost one. Apart from $\mathrm{Gd}$ and $\mathrm{Cr},{ }^{5}$ no other system has yet been found that exhibits this striking property. Several other rare earths, such as $\mathrm{Dy}$ or $\mathrm{Tb}$, should behave similarly to $\mathrm{Gd}$, but their often low bulk critical temperature makes their experimental investigations more difficult.

As the temperature is lowered below the Curie temperature of the surface of $\mathrm{Gd}$, which lies at least ${ }^{2,4} 15 \mathrm{~K}$ above the bulk Curie temperature $T_{\mathrm{C}}^{\text {bulk }}=292.5 \pm 0.3 \mathrm{~K}$ of the bulk, ${ }^{2}$ the surface magnetization increases smoothly. ${ }^{2}$ When the temperature is further lowered and finally reaches the value $T_{\mathrm{C}}^{\text {bulk }}$ at which magnetic order sets in in the bulk, two ferromagnetically ordered phases coexist at equilibrium.

The classical statistical-mechanical description of this phenomenon is well developed, and conventional models of localized magnetism in insulators such as the Heisenberg and Ising models have been analyzed in great detail, although mostly approximately. ${ }^{6}$ As expected, coexistence of an ordered surface phase with a disordered bulk phase is predicted to occur for sufficient enhancement $(10 \%-60 \%$, depending on models and esti- mates) of the interactions between the surface spins. ${ }^{6}$ Simple proofs ${ }^{7}$ of this prediction, based on rigorous correlation inequalities, can be constructed for the Ising and anisotropic $X Y$ (rotator) models; a similar proof for the anisotropic Heisenberg model is still lacking.

Obviously, classical statistical mechanics is unable to explain the origin of the enhancement of the surface coupling, which is purely quantum mechanical. While the proper quantitative theory is, not unexpectedly, extremely difficult and still inexistent, the enhancement of surface couplings is successfully accounted for, qualitatively at least, by combining the theory of itinerant- and localized-electron ferromagnetism. ${ }^{8}$ Indeed, the atoms of the topmost surface layer have fewer neighboring atoms than those of the bulk, and it is well known that magnetic order is strongly modified by the coordination number. Moreover, the changes of the lattice constants near the surface also modify magnetic interactions.

In this Letter, we address the important question of whether and how the singularities of the phase transition of bulk Gd manifest themselves in the behavior of the otherwise regular and noncritical surface magnetization of Gd. While there appears to be no earlier experimental studies of this problem in magnetic systems, a very similar problem has been actively studied in recent times in multiphase classical fluid systems near critical end points ${ }^{9}$ and in the equivalent ${ }^{4} \mathrm{He}$ near the $\lambda$ point. ${ }^{10}$ In these fluid systems, the surface tension has been analyzed in detail and the results, to which we return below, do share several important features with those described here for the surface magnetization of $\mathrm{Gd}$.

The experimental method used to study surface magnetization is based on electron-capture spectroscopy (ECS). The basic process in ECS is the capture of spin-polarized electrons during grazing-angle reflection of fast $(150 \mathrm{keV})$ deuterons at a magnetic surface. For an angle of incidence of $0.2^{\circ}$, the distance of closest ap- 
proach of the deuterons during the specular reflection is $2 \AA$. Thus, the ions probe only the exponential tails of the electronic wave functions at the topmost surface layer, resulting in the extreme surface sensitivity of ECS.

Electron capture at ferromagnetic surfaces results in the formation of deuterium atoms having a net electronspin polarization. By hyperfine interaction, this electronspin polarization is partially converted to a nuclear polarization which provides a direct measure of the net electron-spin polarization $P$ of the electrons captured at the ferromagnetic surface.

In the case of Gd surfaces, as discussed in Ref. 2 and Rau " the measured polarization $P(T, H)$ can be attributed to the polarization of the $5 d-6 s$ surface conduction electrons. It is proportional to surface magnetization $m_{s}(T, H)$ of the localized $4 f$ electrons which are the predominant carriers of the magnetization of $\mathrm{Gd}$ so that $P(T, H)=b m_{s}(T, H)$, where the sign and magnitude of $b$ depends on the spin-polarized $(5 d, 6 s)$-electron density of states at the Fermi level.

The $P(T, H)$ data are obtained for atomically clean and flat magnetized Gd surfaces consisting of a single Weiss domain, as shown by Kerr-effect measurements. With use of scaling theory, the spontaneous polarization $P_{0}(T)$, and therefore the spontaneous magnetization $m_{0}(T)$, of such a single-Weiss-domain specimen is then determined by extrapolation $^{2}$ towards the limit $H \rightarrow 0$. The internal magnetic field was varied between 24 and $64 \mathrm{kA} / \mathrm{m}$, corresponding to a very small internal normalized magnetic field. Further details can be found in Ref. 2.

The experimental data for the spontaneous surface polarization, shown in Fig. 1, give rise to a surface magnetization of the form

$$
m_{S}(T)-m_{s}\left(T_{\mathrm{C}}^{\text {bulk }}\right)=\mathrm{const} \times\left\{\begin{array}{l}
\left(T_{\mathrm{C}}^{\mathrm{bulk}}-T\right)^{x_{-}} / T_{\mathrm{C}}, \text { for } T<T_{\mathrm{C}}^{\text {bulk }}, \\
-\left(T-T_{\mathrm{C}}^{\text {bulk }}\right)^{x_{+}} / T_{\mathrm{C}}, \text { for } T>T_{\mathrm{C}}^{\text {bulk }},
\end{array}\right.
$$

with $x_{-}=1.00 \pm 0.01$ and $x_{+}=1.00 \pm 0.03$. These errors also include the uncertainty on $T_{\mathrm{C}}^{\text {bulk }}$.

Several theoretical predictions have been made concerning the behavior of the nonvanishing surface magnetization at the bulk Curie temperature. It was first shown ${ }^{12}$ that in mean-field approximation, the phenomenological Landau-Ginzburg model predicts that the surface magnetization $m_{s}$ together with its first derivative are continuous functions of temperature at the bulk $\mathrm{Cu}$ rie temperature $T_{\mathrm{C}}^{\text {bulk }}$, but that there is a discontinuity at $T_{\mathrm{C}}^{\text {bulk }}$ in the second derivative of the surface magnetization. In fact, this mean-field approximation is readily solved analytically, yielding, ${ }^{12}$ for $\left|T_{C}^{\text {bulk }}-T\right| \ll 1$,

$$
m_{s}(T)=m_{s}\left(T_{\mathrm{C}}^{\text {bulk }}\right)+A\left(T_{\mathrm{C}}^{\text {bulk }}-T\right)+B_{T}\left(T_{\mathrm{C}}^{\text {bulk }}-T\right)^{2},
$$

where $B_{T}=0$ for $T>T_{\mathrm{C}}^{\mathrm{bulk}}$. We observe that the linear term is also found in the present experiments [Eq. (1)]. We come back to this important point below.

It was suggested ${ }^{13}$ next, on the basis of scaling arguments, renormalization-group considerations, and partly questionable ${ }^{14}$ assumptions, that the singular behavior of the surface magnetization should be identical to that of the bulk free energy. A key assumption underlying this suggestion is that the critical behavior of the surface with enhanced couplings and vanishing surface field is identical to that which obtains in the case of zero surface-coupling enhancement in a nonvanishing surface field. Consequently, because the singular part of the bulk-free-energy carriers, by definition, a critical exponent equal to $2-\alpha$, with $\alpha$ the critical exponent describing the divergence of the bulk specific heat, and because $\alpha<1$, this argument implies that at $T_{C}^{\text {bulk }}$ the surface magnetization and its first temperature derivative are continuous functions of the temperature, but that its second temperature derivative is weakly singular. Stronger singularities have also been suggested. ${ }^{14}$ In mean-field approximation, $\alpha=0$, so that this prediction is consistent with the explicit mean-field system given above.

More generally, it follows from this argument that the expansion of the surface magnetization $m_{s}(T)$ about $T_{\mathrm{C}}^{\text {bulk }}$ in powers of $T_{\mathrm{C}}^{\text {bulk }}-T$ contains a term regular in $T_{\mathrm{C}}^{\text {bulk }}-T$ which, unless it accidentally vanishes altogether, must be linear in $T_{C}^{\text {bulk }}$. Only then is the approximate mean-field result recaptured when $\alpha=0$. It should be noted that the experimental results shown in Fig. 1 clearly rule out a vanishing slope of $m_{s}(T)$ at $T_{\mathrm{C}}^{\text {bulk }}$ and, to- 
gether with the exponent values $x_{ \pm} \sim 1.00$ of (1), are fully consistent with these theoretical predictions.

Clearly, the singularity in the second derivative of the surface magnetization, be it that of the bulk specific heat, in accord with Ref. 13, or a stronger one, as suggested in Ref. 14, is impossible to detect with the eye or experimentally.

Precisely the same qualitative behavior is predicted for the surface tension at a critical end point in multicomponent fluids. In that case, the singularity of the second temperature derivative is predicted to carry a critical exponent equal to $2-\mu$, with $\mu \sim 1.26$. There is, however, an unresolved conflict between theory and experiment for the case of ${ }^{4} \mathrm{He}$, for which extremely accurate experiments ${ }^{15}$ clearly indicate a discontinuity in the first temperature derivative at $T_{\mathrm{C}}^{\text {bulk }}$.

No successful renormalization-group calculation, be it in real space or with the use of the $\epsilon$ expansion, has been performed to determine the detailed singular behavior of the surface magnetization at the bulk Curie temperature. The only result the renormalization-group method has yielded ${ }^{16}$ so far is the meager one that a nonzero surface magnetization exists at $T_{\mathrm{C}}^{\text {bulk }}$.

Theoretical predictions in conflict with those described above have recently been made in a detailed study ${ }^{17}$ of the quantal anisotropic Heisenberg model. This analysis is based on a random-phase approximation of Green's functions. Consequently, unlike the case of a mean-field treatment, spin fluctuations are, to some extent at least, taken into account. The main prediction of this analysis is that while the surface magnetization is continuous at the bulk transition, its first derivative is not. This discontinuity of the first derivative is rather pronounced (see Fig. 1 of Ref. 17), although no precise numerical estimate was given in Ref. 17.

To be sure, the systems considered in Ref. 17, typically EuO, which are semiconductors, differ strongly from the metallic $\mathrm{Gd}$, in which the spin polarization of the $(5 d, 6 s)$ conduction electrons via the Ruderman-KottelKasuya-Yosida interactions may have in general significant effects on the temperature dependence of the magnetization. ${ }^{18}$ But previous experience with critical phenomena indicates that the bulk thermodynamic singularities at critical points of insulating and metallic magnets are identical; experimental tests are unfortunately made difficult by the low Curie temperature ( $T_{\mathrm{C}}^{\text {bulk }}=69.1 \mathrm{~K}$ ) of EuO. It must also be said that the random-phase approximation when applied to ferromagnetism is recognized ${ }^{19}$ to be rather difficult to control, making its quantitative predictions somewhat uncertain.

It would be of great interest to combine the complementary ECS and SPLEED techniques to determine the nature of the magnetic coupling between the first surface layers. At present, the results obtained with ECS and SPLEED suggest that there is antiferromagnetic or at least helical order between the surface layers. However, a definite test of this suggestion is not yet possible because the samples used in SPLEED ${ }^{4}$ are freshly remagnetized at each temperature, whereas no remagnetization is performed in ECS. It is hoped that this first study will stimulate further more detailed theoretical and experimental works.

We are grateful to S. Selzer and N. Majlis for stimulating discussions and valuable correspondence and we thank R. Lipowski, T. C. Lubensky, and G. T. Trammell for instructive conversations. This work was supported by the Shell Oil Company, the Welch Foundation, the National Science Foundation, and the Donors of the Petroleum Research Fund administered by the American Chemical Society.

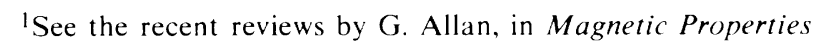
of Low-Dimensional Systems, edited by L. M. Falicov and J. L. Morán-López, Springer Proceedings in Physics Vol. 14 (Springer-Verlag, Berlin, 1986); A. J. Freeman and C. L. Fu, ibid.; M. E. Fisher, Int. J. Quantum Chem. Quantum Chem. Symp. 16, 671 (1982). Older, more detailed reviews are D. L. Mills, J. Phys. (Paris), Colloq. 31, C1-33 (1970); J. C. S. Levy, Surf. Sci. Rep. 1, 39 (1981); G. Allan, Surf. Sci. Rep. 1, 121 (1981).

${ }^{2} \mathrm{C}$. Rau and S. Eichner, in Nuclear Methods in Materials Research, edited by K. Bethge, H. Bauman, H. Jex, and F. Rauch (Vieweg, Braunschweig, 1980), p. 354; C. Rau and S. Eichner, Phys. Rev. B 34, 6347 (1986).

${ }^{3}$ A. Cerri, D. Mauri, and M. Landolt, Phys. Rev. B 27, 6526 (1983).

${ }^{4}$ D. Weller, S. F. Alvarado, W. Gudat, K. Schroder, and M. Campagna, Phys. Rev. Lett. 54, 1555 (1985).

${ }^{5} \mathrm{C}$. Rau and S. Eichner, Phys. Rev. Lett. 47, 939 (1981); G. Allan, Surf. Sci. 74, 79 (1978).

${ }^{6} \mathrm{See}$, for example, the review by K. Binder, in Phase Transitions and Critical Phenomena, edited by C. Domb and J. L. Lebowitz (Academic, London, 1983), p. 1.

${ }^{7}$ D. B. Abraham and C. E. Pfister, Phys. Lett. 96A, 243 (1983).

${ }^{8}$ Allan, Ref. 1; I. Peschel and P. Fulde, Z. Phys. 259, 145 (1973); P. Fulde, Physica (Amsterdam) 91B, 251 (1977).

${ }^{9}$ B. Widom, J. Chem. Phys. 67, 872 (1977); N. Nagarajan, W. W. Webb, and B. Widom, J. Chem. Phys. 77, 5771 (1982); M. Robert and P. Tavan, J. Chem. Phys. 78, 2557 (1983); M. M. Telo da Gamma, R. Evans, and I. Hadjiagapliu, Mol. Phys. 52, 573 (1984); I. Pegg, M. Goh, R. Scott, and C. Knobler, Phys. Rev. Lett. 55, 2320 (1985); J. Lazenby and J. Rudnick, unpublished.

${ }^{10}$ Widom, Ref. 9; J. H. Magerlein and T. M. Sanders, Jr., Phys. Rev. Lett. 36, 258 (1976); P. Tavan and B. Widom, Phys. Rev. B 27, 180 (1983): M. Robert, Helv. Phys. Acta 55, 381 (1982); Robert and Tavan, Ref. 9.

${ }^{11}$ C. Rau, J. Magn. Magn. Mater. 30, 141 (1982), and Ref. 2.

${ }^{12}$ T. C. Lubensky and M. H. Rubin, Phys. Rev. B 12, 3885 (1975). 
${ }^{13}$ A. J. Bray and M. A. Moore, J. Phys. A 10, 1927 (1977).

${ }^{14}$ L. G. Dunfield and J. Noolandi, Phys. Rev. B 22, 2586 (1980).

${ }^{15}$ Magerlein and Sanders, Ref. 10.

${ }^{16} \mathrm{~T}$. W. Burkhardt and E. Eisenriegler, Phys. Rev. B 17, 318

(1978); R. Lipowsky and H. Wagner, Z. Phys. B 42, 355
(1981).

${ }^{17}$ S. Selzer and N. Majlis, Phys. Rev. B 27, 544 (1983).

${ }^{18} \mathrm{~S}$. Selzer and N. Majlis, private communication.

${ }^{19}$ See, for example, S. Doniach and E. H. Sondheimer, Green's Functions for Solid State Physicists (Benjamin, Reading, 1978). 